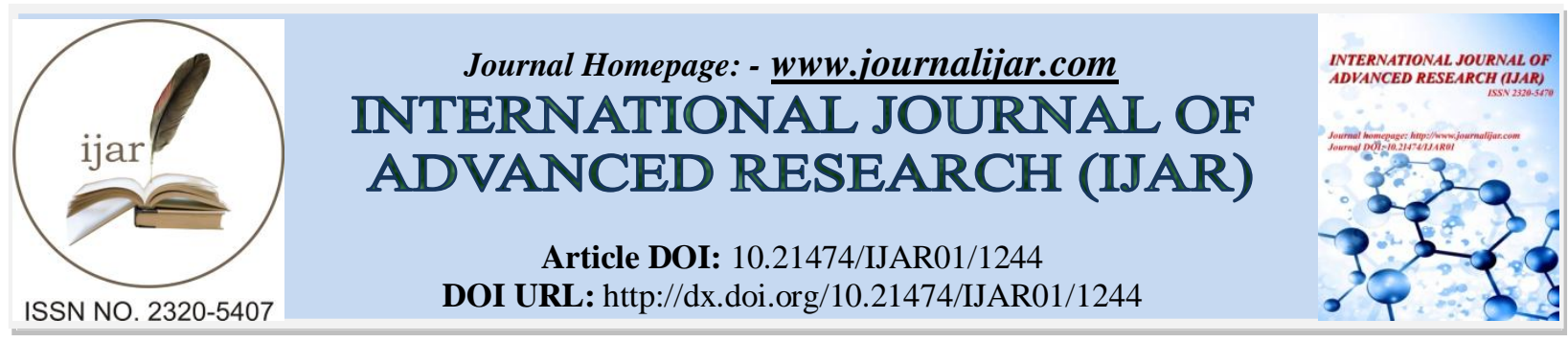

RESEARCH ARTICLE

\title{
Neural Network Radial Basis Function classifier for earthquake data using aFOA
}

\author{
Anurag Rana, Arjun Kumar, Ankur Sharma
}

\section{Manuscript Info}

Manuscript History

Received: 12 June 2016

Final Accepted: 22 July 2016

Published: August 2016

Key words:-

Neural, Network, RBF, aFOA,

Classifier.

\section{Abstract}

Machine Learning is a scientific discipline that is concerned with the design and development of computer programs that automatically improves with experience. Machine learning is the popular classification methods in the field of decision support. To design optimal automated classifier to short out the task of classification is necessary, due to number of parameters. This research was limned the motivation for using Artificial Neural Networks with the assistance of evolutionary swarm algorithm such as fruit fly optimization algorithm to be competent tool in finding the most optimal classifier. Research paper presents NN RBF classifier for earthquake data. Proposed Amended Fruit Fly optimization technique improves the performance of classifiers classification accuracy. Research paper analyses and design an optimal classifier using Radial Basis Function Neural Nets.

Copy Right, IJAR, 2016,. All rights reserved.

\section{Introduction:-}

Classification is a decision-making task for real world problems. When an object requires to be classified into a predefined class on the basis of attributes of the object, the classification will be used. Credit risk evaluation, speech recognitions, handwritten character recognition, bankruptcy prediction and quality control [1]. Binary and multiclass problems are two types of classification problems. The outcome of prediction has to be determined with decision of yes or no, is a binary problem. And, in multiclass problem, predicted result determined as multiple outcomes [2]. Artificial Neural Network (ANN), Classification trees and support vector machines (SVM) are used to solve classification problems. Logistic Regression discriminate analysis, linear regression, fuzzy logic, Bayesian network, k-mean clustering technique and genetic algorithms [3] are examples of classification problems solving techniques. Fuzzy probabilistic neural network [7], recursive partitioning of the majority class algorithm [6] and neuro-fuzzy based classification technique [5] are hybrid techniques have been implemented.

In this paper, a design of optimal classifier for classification of strong ground motion data has been analyzed using Neural Nets with aFOA.

\section{Related work:-}

Number of techniques proposed to perform classification. Classification problem is a decision making job where many research have been working on. In artificial intelligence, neural network is the successful examples to solve classification problem.

In paper [9] the author use Perceptron, back-propagation network and probabilistic neural network classification algorithm based on artificial neural network. 
In research paper [10] the researcher presents neural network techniques comparison for binary classification problems. For comparison are back propagation Neural network (BPNN), Radial basis function Neural Network (RBFNN), General Regression Neural Network (GRNN), probabilistic Neural Network (PNN) and complementary Neural Network (CMTNN). The classification performance obtained by five different types of neural networks and comparison based on three benchmark datasets obtained from machine learning repository.

In paper [11] author design classifier using MLP NN trained with back propagation algorithm. Using the first 200 instances for training which were carefully split almost 50\% positive and 50\% negative, they found that MLP NN trained standard back propagation algorithm.

Literature review state that for the multilayer preceptron (MLP) NN trained with back propagation reported average classification accuracy was about $82 \%$ on the test instances. This paper proposed NN RBF with aFOA technique for classification.

\section{Proposed method:-}

\section{Neural network radial basis function:-}

NN RBF a nearest neighbor classifier. It uses Gaussian transfer functions having radial symmetry [13]. The centers and widths of the Gaussian (radial basis functions) are set by unsupervised learning rules, and supervised learning is applied to (cluster centers), because it is problem dependent. The behavior of any arbitrary function $f(x)$ is described in a compact areas of the input space, by a combination of simpler functions

$$
\hat{O} \mathrm{i}(\mathrm{x})=\mathrm{y}(\mathrm{x}-\mathrm{xi} !) \text {, }
$$

where $y($.$) is normally a Gaussian function. The appropriate to the function f(x)$ is given as $f(x, w)=$ ? wi $G(! x-$ xi!), where wi are real value entries of the coefficient vector

$f(x)$ being a real valued vector

$$
\mathrm{w}=[\mathrm{w} 1, \mathrm{w} 2, \mathrm{w} 3-, \mathrm{wn}],
$$

$$
\mathrm{x}=[\mathrm{x} 1, \mathrm{x} 2, \mathrm{x} 3-, \mathrm{xn}],
$$

implements the input -output map of the NN RBF . Any arbitrary continuous function can be approximated with an NN RBF if localized Gaussian are placed to cover the space, the width of each Gaussian is controlled, the amplitude of each Gaussian is set.

Amended fruit fly optimization:-

According to food finding characteristics of fruit fly swarm, the whole procedure of the original FOA [12] can be divided into several step as follows:-

\section{Algorithm for aFOA:- \\ Step 1 Start:- \\ Start the algorithm.}

Step 2 Parameter Initializations:-

The main parameters of aFOA are maximum iteration number, the population size.

Step 3 Population Initializations:-

Randomly initialize the population size is NP. Randomly produce solution, half blending and un-blending.

Step 4 Neighborhood Generations and Find Best Neighborhood:-

NN neighborhoods are randomly generated. NN neighborhoods are produced by three kinds of neighborhood search. Find out the best neighbor.

Step 5 Replacements of Neighbor:-

If the best neighbor is better than the fly then replace the fly with the best neighbor and go to next step, else go to next step without replacing.

Step 6 Local Neighborhood Loop Search:-

If the loop termination is reached then sort the population else go back to step 4.

Step 7 Crossovers:

In global cooperation search each flies in the poor half crossover it with the corresponding one. If new fly is better than poor fly then replace the poor fly with new one and go to next step, else go to next step without replacing.

Step 8 Termination Criterions:- 
If termination standard is reached then provide result, else go back to step 3.

Step 9 End:-

End the algorithm.

\section{Result \& discussions:-}

NN RBF is designed using variable parameters, number of cluster centers (Gaussian basis functions), competitive learning rule, used in unsupervised competitive learning, transfer function of neurons in o/p layer, learning rule used in the o/p layer ,maximum number of epoch used for supervised learning and error threshold for training dataset. The chosen optimal configuration of the NN RBF is trained three times with 100 epochs in unsupervised learning \& 5000 epochs in supervised learning. Select 100 of no. of cluster centers, conjugate gradient supervised learning rule,tanh output layer transfer function and Euclidean competitive learning matrix. Simulation result of NN RBF classifier gives $95.59 \%$ accuracy.

\section{Conclusion:-}

We implemented RBF Neural network classifiers for classification of radar data. For the classification of seismograph returns from the ionosphere, the decision boundaries form by the NN RBF classifier are seen to be more accurate. The main novelty of this paper is in the proposed aFOA-based approach, which aims at optimizing the performances of NN classifier in terms of classification accuracy. In addition, we explored various other implementations such as Support Vector Machine with aFOA \& empirically select this particular network since it achieved the best performance.

\section{Acknowledgement:-}

I THANKS TO MY PARENTS , MY WIFE PREETI CHIB RANA (MCA) FOR THEIR CONTINOUS SUPPORT. I THANKS BRIG. S.C. VERMA (V.C ARNI UNIVERSITY) FOR MOTIVATING AND PROVIDEING STRONG CO-OPERATION. I ALSO THANKS TO ALL SUPPORTING STAFF.

\section{References:-}

1. G .P.Zhange "Neural networks for classification: a survey," systems, Man, and cybernetics, part C:Application and Reviews, IEEE Transaction on,vol.30.pp.451-462.2000.

2. P.Kraipeerapun,"Neural network classification based on quantification of uncertainty",Murdoch University, 2008.

3. S.B.Kotsiantis,"Supersed Machine learning: A review of classification techniques", informatics, vol.31. pp 24268, 2007.

4. Y.Sun, F.Karray,and Al-sharhan."Hybrid soft computing techniques for heterogeneous data classification." In fuzzy systems, 2002, fuzz-IEEE02.

5. H.Ahumada,G.L.Grinblat,L.C.Uzal,P.M.Granitto,and .ceccatto,” REPMAC: Anew hybrid intelligent systems 2008.

6. Y.Huang and C,Tian "Research on credit risk assessment model of commercial banks based on fuzzy probabilistic neural network" in risk management \& engineering management,2008

7. Sigillito Vince (1989). Applied physics laboratory, john Hopkins university Ionosphere database.

8. X iao-Feng Gu \& Lin Liu,Yuan-Yuan Huang (2008). Data classification based on Artificial Neural Networks. IEEE 2008.

9. P.Jeatrakul and K.W.Wong; "Comparing the performance of different Neural Networks for Binary classification problems" Natural language processing 2009 SNL P-09, eight international symposium,111-115,IEEE 2009.

10. Sigillito.V.G,Wing,S.P.Hutton,L.V..Baker,K.B(1989)"Classification of radar returns from the ionosphere using neural networks" John Hopkins APL technical digest, vol 10 pp 262-266.

11. Chanjun Zhu,Bin Wang;"PSO-based RBF Neural Network Model for Teaching Quality Evalution"2009 IEEE,41-50,CASE-2009 IITA .

12. Anurag Rana and Ankur Sharma; "Optimization of Radial Basis Neural Network by mean of Amended Fruit Fly Optimization Algorithm" Journal of Computer and Mathematical Sciences, Abbr: J.Comp. \& Math. Sci. 2014, Vol.5(3): pg.262-272. 
13. Anurag Rana and Ankur Sharma; "Resolving Set-Streaming Stream-Shop Scheduling in Distributed System by mean of an aFOA" International Journal of Computer Science \& Engineering Technology (IJCSET) Vol. 5 No. 04 Apr 2014.

14. Arjun Kumar, Ashwani Kumar, Ashok Kumar, Himanshu Mittal and Rakhi Bhardwaj (2012). Software to Estimate Spectral and Source Parameters. International Journal of Geosciences, 3(5), 1142-1149.

15. Ashwani Kumar, Arjun Kumar, S. C. Gupta, Himanshu Mittal and Rohtash Kumar (2013). Source Parameters and $f_{\max }$ in Kameng Region of Arunachal Lesser Himalaya. Journal of Asian Earth Sciences, 70-71, 35-44.

16. Ashwani Kumar, Arjun Kumar, S. C. Gupta, A. K. Jindal and Vandana Ghangas (2014). Source Parameters of Local Earthquakes in Bilaspur Region of Himachal Lesser Himalaya. Arabian Journal of Geosciences, 7(6), 2257-2267. 